\title{
A 6-Year-Old Girl with Recurrent Rhabdomyolysis
}

\section{Cátia Vilas Boas Leitão ${ }^{1 *}$, Isabel Ayres Pereira ${ }^{1}$, Joana Tenente ${ }^{1}$ and Helena Santos ${ }^{2}$}

${ }^{1}$ Pediatrics Department, Unidade 2, Centro Hospitalar Vila Nova de Gaia/Espinho, Portugal

${ }^{2}$ Metabolic Disorders, Child and Adolescent Neurosciences Unit, Pediatrics Department, Centro Hospitalar Vila Nova de Gaia/Espinho, Portugal

*Corresponding Author: Cátia Vilas Boas Leitão, Pediatrics Department, Unidade 2, Centro Hospitalar Vila Nova de Gaia/Espinho, Portugal.

DOI: 10.31080/ASPE.2020.03.0211
Received: January 18, 2020;

Published: January 29, 2020

(C) All rights are reserved by Cátia Vilas Boas Leitão., et al.

\begin{abstract}
Rhabdomyolysis is the ocorrence of muscular necrosis with release of its constituents into the circulation and can be a manifestation of multiple disorders. In this case report, a 6-year-old female patient presented to the emergency department with a history suggestive of rhabdomyolysis in context of EBV infection. The patient was later reevaluated and an accurate previous medical history and follow-up lead to the final diagnose of an uncommon neurometabolic pathology - McArdle disease or type $\mathrm{V}$ glycogen storage disease. This case highlights the importance of investigating rhabdomyolysis' possible etiologies, in order to allow an early diagnosis and prevent acute decompensations.
\end{abstract}

Keywords: Child; McArdle Disease; Rhabdomyolyis; Metabolic

\section{Abbreviations}

CK: Creatine Kinase; EBV: Epstein Barr Virus; GSD: Glycogen Storage Diseases

\section{Case Report}

A 6-year-old caucasian girl was observed in the emergency department for fever, odynophagia, anorexia and myalgia. She had past history of elevated creatine kinase $(\mathrm{CK})$ and toe walking till the age of 2, with intermittent CK elevation, and otherwise normal physical examination and basic etiological investigations. She had no other relevant personal or family history, and a normal newborn expanded metabolic screening.

At physical examination she was well-appearing but febrile (temperature of $39.3^{\circ} \mathrm{C}$ ), with dry lips, enanthema, conjunctival hyperemia, bilateral cervical adenopathies and muscular pain. Blood tests revealed mild anemia (hemoglobin level, $11.4 \mathrm{~g} / \mathrm{dL}$ ), and elevation of white blood cell count (12530/uL with $47.7 \%$ lymphocytes), CK (2196 U/L), lactate dehydrogenase (649 U/L) and C-reactive protein $(1,97 \mathrm{mg} / \mathrm{dl})$. Liver enzymes, urea and creatinine values were normal, as well as urine biochemistry. A rapid Streptococcus pyogenes diagnostic test was negative. Blood serol- ogy showed positive Epstein Barr Virus (EBV) IgM and negative IgG, in favor of acute EBV infection. The girl was discharged with symptomatic treatment for presumptive diagnosis of rhabdomyolysis associated to EBV infection.

The patient was reassessed on pediatric outpatient department two weeks later, and a past history of myalgia and exercise intolerance was found. A gastrocnemius hypertrophy was noted, with no other alterations on the physical and neurological examinations. Gowers sign was negative. Over the next several months CK levels were monitored, fluctuating between 400 and $8250 \mathrm{U} / \mathrm{L}$. Investigations for metabolic causes of rhabdomyolysis, including dry blood spot acylcarnitines, blood amino acids, lactate and pyruvate and urine organic acids were normal.

To clarify the rhabdomyolysis etiology a muscle biopsy was performed and revealed glycogen deposits and an absence of myofosforilasis, suggestive of McArdle disease. The diagnosis was confirmed by the presence of c.148C $>\mathrm{T}$ (p.Arg50*) and c.40delA (p.Ile14Serfs*12) variants in PYGM gene.

Patient was started on a carbohydrate enriched diet and light to moderate aerobic exercise regimen, and information on rhabdo- 
myolysis was given. After two years, a progressive normalization of CK levels and improvement of physical activity tolerance was observed. No further rhabdomyolysis episodes were documented.

\section{Discussion}

Rhabdomyolysis is a life-threatening condition characterized by rapid breakdown and leakage of skeletal muscle cell contents, such as electrolytes, myoglobin, and other sarcoplasmic proteins (e.g., CK, aldolase, lactate dehydrogenase, alanine aminotransferase, and aspartate aminotransferase) into the circulation [1-5]. It is a well known condition but there is not an international consensus for its definition $[1,3]$. A recent systematic review describes it as a syndrome of acute muscle weakness, myalgia, and swelling combined with a CK cut-off value of $>1000$ IU/L or CK $>5 \mathrm{X}$ upper limit of normal (for the standard definition of a mild rhabdomyolysis). The presence of myoglobinuria and acute renal failure signals a severe case [3].

A number of etiologies have been identified, that can be categorized into acquired and inherited causes. Regarding acquired causes, the most common in children are infection (viral myositis are responsible for up to one third of the cases), physical exertion, inflammation, trauma and myotoxins $[1,2,4,5]$.

Inherited muscle disorders that predispose to rhabdomyolysis in children can be divided in three major groups: metabolic myopathies, muscular dystrophies and congenital myopathies (table 1) $[4,5]$. Clinical manifestations are variable, ranging from permanent symptoms such as progressive weakness, to episodic symptoms of exercise intolerance, myalgia and/or myoglobinuria [6,7].

Metabolic myopathies, are a heterogeneous group of disorders usually divided into three main categories: glycogen storage diseases (GSD), fatty acid metabolism defects and mitochondrial disorders [4-6,8] (see table 1). Despite representing a very small percentage, this etiology must be kept in mind, mainly in patients with recurrent episodes after exertion or infection [6].

In this case, an accurate previous medical history and follow-up lead to the final diagnosis. The past history of gait abnormalities, myalgia and exercise intolerance associated with high CK levels and recurrent episodes of rhabdomyolysis were consistent with the presence of an underlying myopathic disorder.

As this patient presented clinical signs and symptoms suggestive of an underlying muscular disorder, and previous metabolic workup was normal, a muscle biopsy was performed and sowed alterations, suggestive of McArdle disease. Genetic test for PYGM sequencing confirmed the diagnosis of McArdle disease - type $\mathrm{V}$ glycogen storage disease.

\begin{tabular}{|c|c|}
\hline \multirow[t]{21}{*}{$\begin{array}{l}\text { Metabolic } \\
\text { myopathies }\end{array}$} & $\begin{array}{l}\text { Glycogen storage diseases } \\
\text { McArdle disease, myophosphorylase deficiency } \\
\text { (GSD V) }\end{array}$ \\
\hline & $\begin{array}{l}\text { Tarui disease, phosphofructokinase deficiency (GSD } \\
\text { VII) }\end{array}$ \\
\hline & Phosphorylase b kinase deficiency (GSD IX) \\
\hline & Phosphoglycerate mutase deficiency (GSD X) \\
\hline & Lactate dehydrogenase deficiency (GSD XI) \\
\hline & Debrancher enzyme deficiency (GSD III) \\
\hline & $\beta$-Enolase deficiency (GSD XIII) \\
\hline & Glycogen synthase deficiency \\
\hline & Phosphoglucomutase deficiency \\
\hline & Phosphoglycerate kinase 1 deficiency \\
\hline & Fatty acid metabolism defects \\
\hline & Carnitine-palmitoyl-transferase deficiency \\
\hline & Carnitine deficiency \\
\hline & Short and very long-chain acyl-CoA dehydrogenase \\
\hline & Mitochondrial disorders \\
\hline & $\begin{array}{l}\text { Mitochondrial encephalomyopathy, lactic acidosis, } \\
\text { and stroke like episodes (MELAS) due to mitochon- } \\
\text { drial tRNA point mutation }\end{array}$ \\
\hline & Cytochrome-b-oxidase deficiency \\
\hline & Cytochrome-c-oxidase deficiency \\
\hline & Others: \\
\hline & Lipin-1 deficiency \\
\hline & Myoadenylate deaminase deficiency \\
\hline \multirow{6}{*}{$\begin{array}{l}\text { Muscular } \\
\text { dystrophies }\end{array}$} & Duchenne muscular dystrophy \\
\hline & Becker muscular dystrophy \\
\hline & Fukutin-related proteinopathy \\
\hline & Dysferlinopathy \\
\hline & Anoctaminopathy \\
\hline & Sarcoglycanopathy \\
\hline \multirow[t]{2}{*}{$\begin{array}{l}\text { Congenital } \\
\text { myopathies }\end{array}$} & $\begin{array}{l}\text { Malignant hyperthermia susceptibility caused by } \\
\text { ryanodine receptor } 1 \text { gene mutation }\end{array}$ \\
\hline & Selenoprotein N related Myopathies \\
\hline
\end{tabular}

Table 1: Inherited causes of rhabdomyolysis in children $[4,5,8]$.

McArdle disease, first described in 1951, is a metabolic myopathy and one of the most common causes of abnormal muscle glycogenosis [6,8-10]. It is an autosomal recessive disorder caused by PYGM pathogenic variants, causing a myophosphorylase deficiency, with an estimated incidence of $1 / 100,000$ [6,11-13].

Presentation varies from early childhood to adult age, with some rare cases of late-onset disease ( $>60$ years-old) [12]. Only $4 \%$ of cases are diagnosed before the age of ten, because affected children are often considered to be just less active $[9,11,13]$. 
Usually symptoms include weakness, myalgia, cramps and exercise intolerance, with stiffness of exercising muscles, relieved by rest [8-12]. A classical symptom is the "second wind phenomenon". Some minutes after the onset of the typical exercise-induced myalgia, with the continuation of the physical activity, the patients report a decrease in the level of effort, pain and fatigue. This phenomenon is explained by the shift of the metabolic pathway used to generate energy, using fatty acids to provide enough energy to continue the exercise $[5,8,9,11]$.

Neurological evaluation is usually normal between crises, but proximal muscle weakness and wasting occur in approximately $35 \%$ of patients over 40 years of age [9]. A hypertrophied gastrocnemius and an atrophy of the girdle muscles are secondary signs of this disease [11].

Although this is not a life-threatening condition, severe stress on the muscle can have serious consequences. During episodes of rhabdomyolysis, CK levels can increase to more than 100.0001.000.000 U/L, with a high risk of myoglobinuria resulting in acute renal failure and hyperkaliemia that can cause life threatening dyschromia's $[8,11]$.

Diagnosis should be made as early as possible and professionals should be aware of some clinical clues, like exercise-induced symptoms and the "second wind phenomenon" (which is pathognomonic). Diagnostic investigations may include: 1-non-ischemic exercise test, which has a high sensibility for GSD V disease - venous ammonia levels should be assessed in parallel with lactate levels - an abnormal increase in ammonia accompanied by a normal or minimally increased lactate level may be suggestive. Severe serum CK elevation (usually greater than $1000 \mathrm{U} / \mathrm{L}$ at rest) may be seen. 2- Muscle biopsy usually confirms the diagnosis, with the usual finding of vacuolar subsarcolemmal accumulation of glycogen (normally digested by diastase), and negative staining for myophosphorylase [6,9]. 3 - Genetic testing for pathogenic variants in the PYGM gene typically supports the final diagnosis and can obviate the need for muscle biopsy and non-ischemic exercise test if the clinical suspicion is high [9]. Electromyography does not allow for a specific diagnostic, and therefore is not mandatory for the diagnosis $[9,10]$.

At the present time, there is no specific etiological treatment. The prognosis is good and the most important general recommendations include diet and lifestyle modifications [6,8-13]. Exercise intolerance may be alleviated by aerobic conditioning programs, with low to moderate intensity regular aerobic exercise $[9,12,13]$. A carbohydrate-rich diet and low doses of protein may be of benefit for patients with myophosphorylase deficiency $[10,12]$. Oral sucrose taken 5 to 15 minutes before planned activity may have a prophylactic effect [8-10,13]. Patients should avoid strenuous ef- forts and leisure activities that put them at risk and may precipitate rhabdomyolysis and myoglobinuria, such as swimming far from the shore and mountaineering $[9,13]$. Other treatment options described in the literature are supplementation with creatine, vitamin B6 and coenzyme Q10 [13,14].

During an episode of severe rhabdomyolysis, the patient should be hospitalized and undergo hyperhydration with intravenous caloric fluids [6].

\section{Conclusion}

Rhabdomyolysis is frequent in childhood, and can be a manifestation of multiple disorders, most frequently transient and benign. However, recurrent rhabdomyolysis should raise the suspicion of an underlying muscular disorder and should be investigated.

Metabolic myopathies are rare but should be considered in case of recurrent rhabdomyolysis, myalgias, weakness or exercise-induced fatigue [10].

A timely diagnosis of metabolic myopathy is crucial for detection and prevention of acute decompensations, morbidity and renal failure.

\section{Acknowledgements}

We would like to thank Dr. Jorge Sales Marques, Dr. Marta Vila Real, Dr. Margarida Reis Lima and Dr. Manuel Melo Pires for their contribution to this case report.

\section{Conflict of Interest}

The authors declare there is no financial interest or any conflict of interest in conducting this work.

\section{Bibliography}

1. Park Y., et al. "Clinical Characteristics of Rhabdomyolysis in Children: Single Center Experience". Childhood Kidney Diseases 22.2 (2018): 52-57.

2. Elsayed EF and Reilly RF. "Rhabdomyolysis: a review, with emphasis on the pediatric population". Pediatric Nephrology 25.1 (2010): 7-18.

3. Stahl K., et al. "A systematic review on the definition of rhabdomyolysis". Journal of Neurology (2019).

4. Zutt R., et al. "Rhabdomyolysis: review of the literature". Neuromuscular Disorder 24.8 (2014): 651-659.

5. Chan EK., et al. "A diagnostic approach to recurrent myalgia and rhabdomyolysis in children". Archives of Diseases in Childhood (2015): 1-5.

6. Condés R., et al. "Miopatias metabólicas". Revista Medica clinica las Condes 29.6 (2018) 622-635. 
7. Zhao X., et al. "McArdle disease: a "pediatric" disorder present in an adult with acute kidney injury". Clinical and Experimental Nephrology (CEN) Case Reports 6.2 (2017): 156-160.

8. Tarnopolsky MA. "Metabolic Myopathies". Continuum (Minneap Minn) 22.6 (2016): 1829-1851.

9. Saudubray JM., et al. "Inborn Metabolic Diseases - Diagnosis and Treatment”. 6th Edition. Springer, Berlin 5 (2016): 130131.

10. Keel B and Brit M. "McArdle's Disease: a Clinical Review and Case Report". Tennesse Medicine: Journal of the Tennesse Medical Association 106.10 (2013): 33-37.

11. Pomarino D., et al. "McArdle's disease: A differential diagnosis of idiopathic toe walking". Journal of Orthopaedics 15.2 (2018): 685-689.

12. Krishnamoorthy N., et al. "Glycogen storage disease type V (McArdle's disease): a report on three cases". Neurology India 59.6 (2011): 884-886.

13. Bollig G. "McArdle's disease (glycogen storage disease type V) and anesthesia--a case report and review of the literature". Paediatric Anaesthesia 23.9 (2013): 817-823.

14. Sato S., et al. "Confirmation of the efficacy of vitamin B6 supplementation for McArdle disease by follow-up muscle biopsy". Muscle Nerve 45.3 (2012): 436-440.

\section{Assets from publication with us}

- Prompt Acknowledgement after receiving the article

- Thorough Double blinded peer review

- Rapid Publication

- Issue of Publication Certificate

- High visibility of your Published work Website: www.actascientific.com/

Submit Article: www.actascientific.com/submission.php Email us: editor@actascientific.com

Contact us: +919182824667 